\title{
The Application of a Black-Box Solver with Error Estimate to Different Systems of PDEs
}

Torsten Adolph and Willi Schönauer

Forschungszentrum Karlsruhe

Institute for Scientific Computing

Karlsruhe, Germany

\{torsten.adolph, willi.schoenauer\}@iwr.fzk.de

http://www.fzk.de/iwr

http://www.rz.uni-karlsruhe.de/rz/docs/FDEM/Literatur 


\section{Forschungszentrum Karlsruhe}

in der Helmholtz-Gemeinschaft

\section{Motivation}

Numerical solution of non-linear systems of Partial Differential Equations (PDEs)

- Finite Difference Method (FDM)

- Finite Element Method (FEM)

- Finite Volume Method (FVM)

\section{Finite Difference Element Method (FDEM)}

Combination of advantages of FDM and FEM:

FDM on unstructured FEM grid

Supported by: German Ministry of Research (BMBF)

Research Alliance Fuel Cells of Baden-Wuerttemberg 


\section{Objectives}

- Elliptic and parabolic non-linear systems of PDEs

- 2-D and 3-D with arbitrary geometry

- Arbitrary non-linear boundary conditions (BCs)

- Subdomains with different PDEs

- Robustness

- Black-box (PDEs/BCs and domain)

- Error estimate

- Order control/Mesh refinement

- Efficient parallelization (on distributed memory parallel computers) 


\section{Difference formulas of order $q$ on unstructured grid}

Polynomial approach of order $q$ ( $m$ coefficients)

2-D: $m=(q+1) \cdot(q+2) / 2$

3-D: $m=(q+1) \cdot(q+2) \cdot(q+3) / 6$
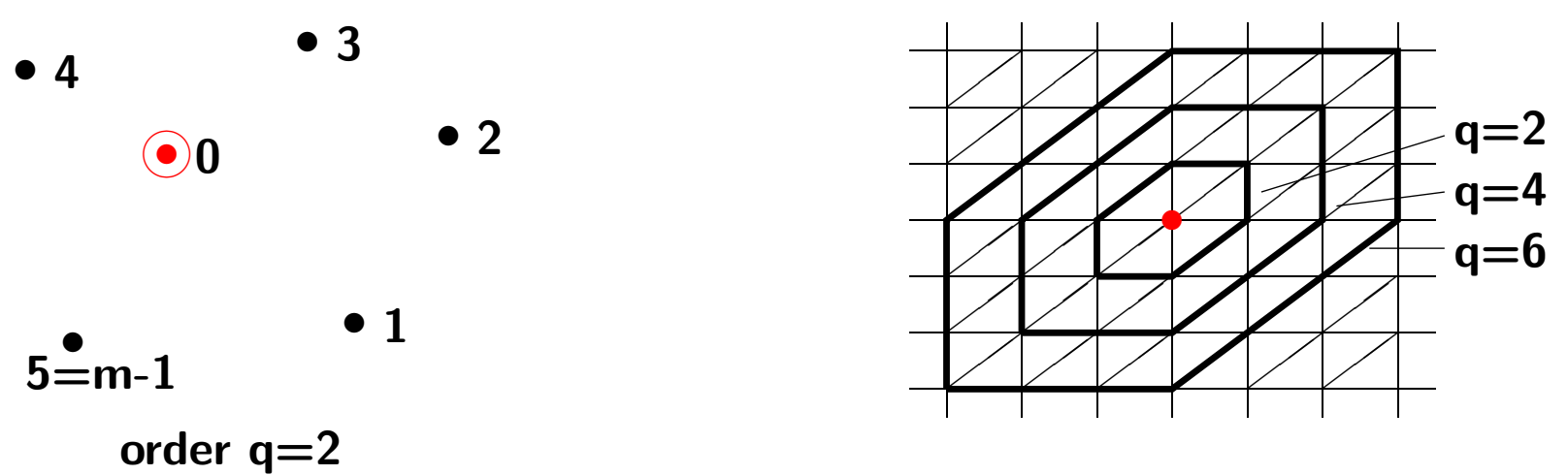

Influence polynomial $P_{q, i}=\left\{\begin{array}{ll}1, & \text { node } \mathrm{i} \\ 0, & \text { other nodes }\end{array} \rightarrow u_{d}, u_{x, d}, u_{y, d}, u_{x x, d}, u_{y y, d}, u_{x y, d}\right.$

Search for nodes in rings (up to order $q+\Delta q$ ) $\rightarrow \mathbf{m}+\mathbf{r}$ nodes

Selection of $\mathbf{m}$ appropriate nodes by special sophisticated algorithm 


\section{Discretization error estimate}

e.g. for $u_{x}: \quad u_{x}=u_{x, d, q}+\bar{d}_{x, q}=u_{x, d, q+2}+\bar{d}_{x, q+2}$

$$
\rightarrow d_{x, q}=u_{x, d, q+2}-u_{x, d, q}\left\{+\bar{d}_{x, q+2}\right\}
$$

\section{Error equation}

$P u \equiv P\left(t, x, y, u, u_{t}, u_{x}, u_{y}, u_{x x}, u_{y y}, u_{x y}\right)$

Linearization by Newton-Raphson

Discretization with error estimates $d_{t}, d_{x}, \ldots$ and linearization in $d_{t}, d_{x}, \ldots$

$$
\begin{aligned}
& \rightarrow \Delta u_{d}=\quad \Delta u_{P u}+\Delta u_{D_{t}}+\Delta u_{D_{x}}+\Delta u_{D_{y}}+\Delta u_{D_{x y}}=\quad \text { (level of solution) } \\
& =Q_{d}^{-1} \cdot\left[(P u)_{d}+D_{t}+\left\{D_{x}+D_{y}+D_{x y}\right\}\right] \quad \text { (level of equation) }
\end{aligned}
$$

Only apply Newton correction $\Delta u_{P u}$ :

$\rightarrow Q_{d} \cdot \Delta u_{P u}=(P u)_{d} \quad$ (computed by LINSOL, Univ. of Karlsruhe)

Other errors for error control and error estimate 


\section{Academic Examples}

Generate "test PDE" from original PDE for given solution $P \bar{u}: P u=0 \Rightarrow P u-P \bar{u}=0$

PDE system:

$$
\begin{aligned}
u_{x x}+u_{y y}+\omega_{y}-f_{1} & =0 \\
v_{x x}+v_{y y}-\omega_{x}-f_{2} & =0 \\
u \omega_{x}+v \omega_{y}-\left(\omega_{x x}+\omega_{y y}\right) / R e-f_{3} & =0, \quad R e=1
\end{aligned}
$$

Boundary conditions: $u-g_{1}=0, v-g_{2}=0, \quad \omega+u_{y}-v_{x}-g_{3}=0$

Test solution: $\quad$ Sugar loaf type function $\bar{u}=e^{-32\left(x^{2}+y^{2}\right)}$

Solution domain: Unit circle with 751 nodes, 1410 elements

Self-adaptation: Mesh refinement and order control, tol $=0.25 \%$

\begin{tabular}{cccccccccc}
\hline & \multirow{2}{*}{$\begin{array}{c}\text { no. } \\
\text { of }\end{array}$} & \multirow{2}{*}{$\begin{array}{c}\text { no. } \\
\text { of }\end{array}$} & $\begin{array}{c}\text { no. of } \\
\text { ref. }\end{array}$ & \multicolumn{2}{c}{$\begin{array}{c}\text { no. of nodes } \\
\text { with order }\end{array}$} & \multicolumn{2}{c}{$\begin{array}{c}\text { global relat. } \\
\text { error }\end{array}$} & \multirow{2}{*}{$\begin{array}{c}\text { sec. } \\
\text { for }\end{array}$} \\
\cline { 7 - 10 } nodes & elem. & nodes & 2 & 4 & 6 & exact & estimated & cycle \\
\hline 1 & 751 & 1410 & 132 & 427 & 320 & 4 & $0.305 E-01$ & $0.280 \mathrm{E}-01$ & 1.021 \\
\hline 2 & 1332 & 2493 & 345 & 180 & 1144 & 8 & $0.109 \mathrm{E}-01$ & $0.950 \mathrm{E}-02$ & 3.604 \\
\hline 3 & 2941 & 5469 & - & 360 & 2556 & 25 & $0.179 \mathrm{E}-02$ & $0.174 \mathrm{E}-02$ & 10.086 \\
\hline
\end{tabular}

HP XC6000, Univ. of Karlsruhe, $1500 \mathrm{MHz}$ Itanium2 core, 6000 MFLOPS peak, np=8 processors 


\section{Forschungszentrum Karlsruhe}

in der Helmholtz-Gemeinschaft

\section{Academic Examples (continued)}

Test solution $\bar{u}=e^{-32\left(x^{2}+y^{2}\right)}$
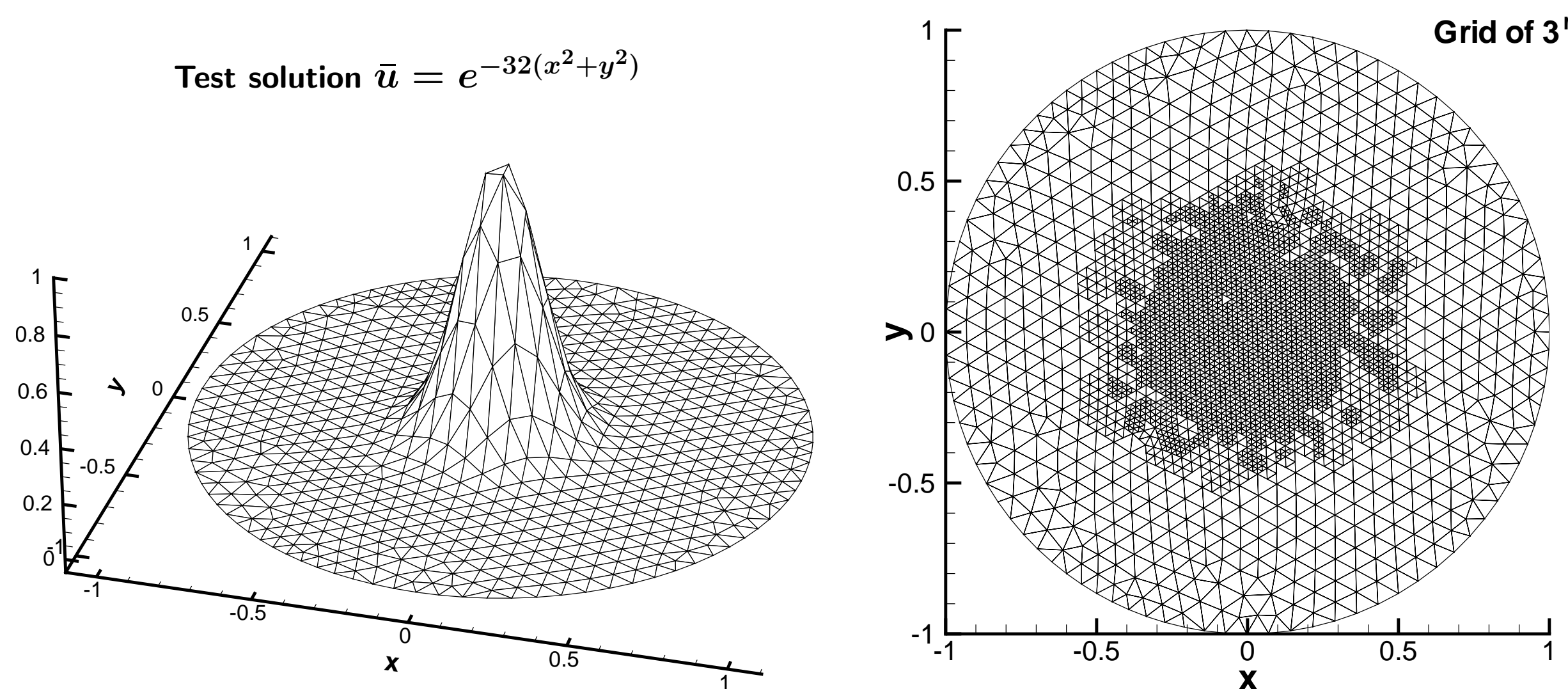


\section{Numerical Simulation of PEM Fuel Cells}

Domain of solution: Gas diffusion layer (GDL)

Variables: Molar flux densities of oxygen, water vapour and nitrogen in $x$ - and $y$-direction, partial pressures for oxygen, water vapour and nitrogen, total pressure and current density

Nonlinear system of 11 PDEs

Rectangular grid with $200 \times 201$ nodes

Consistency order $q=4$

HP XC6000 with 32 processors

CPU time for master processor: $4123 \mathrm{sec}$

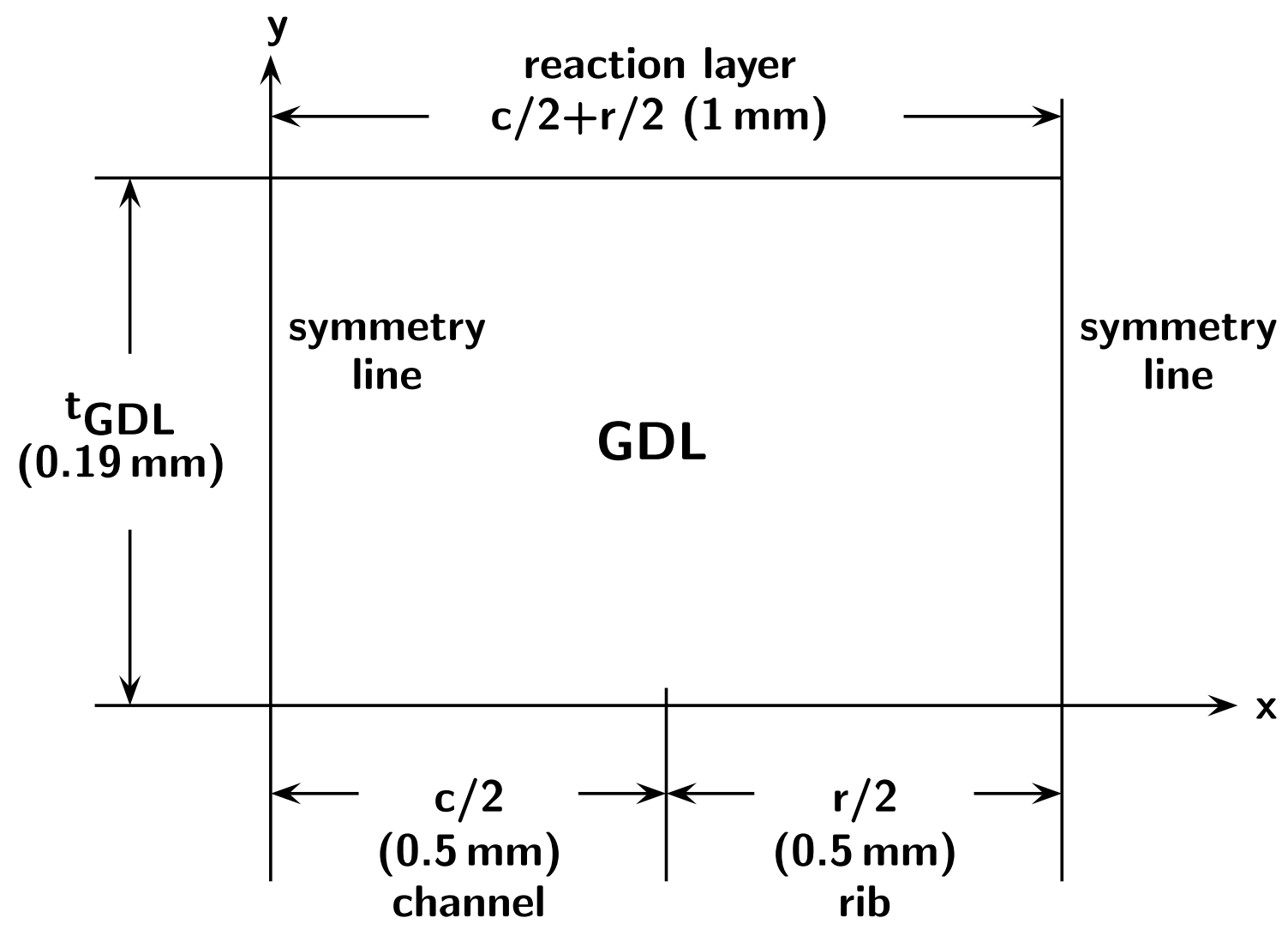




\section{Forschungszentrum Karlsruhe} in der Helmholtz-Gemeinschaft

\section{Numerical Simulation of PEM Fuel Cells (continued)}

6 transport equations

$$
\begin{gathered}
\frac{\dot{n}_{o}^{x}}{D K n_{o}}+\frac{p_{w} \dot{n}_{o}^{x}-p_{o} \dot{n}_{w}^{x}}{D_{o w} \cdot p}+\frac{p_{n} \dot{n}_{o}^{x}-p_{o} \dot{n}_{n}^{x}}{D_{o n} \cdot p}=-\frac{1}{R T} \frac{\partial p_{o}}{\partial x}+\frac{p_{o}}{R T p} \frac{\partial p}{\partial x}-\left[\frac{B_{o}}{D K n_{o}}+\frac{B_{o} p_{w}}{D_{o w} \cdot p}\left(1-\frac{B_{w}}{B_{o}}\right)+\frac{B_{o} p_{n}}{D_{o n} \cdot p}\left(1-\frac{B_{n}}{B_{o}}\right)\right] \frac{p_{o}}{R T p} \frac{\partial p}{\partial x} \\
B_{\nu}=B_{\nu}\left(p_{o}, p_{w}, p_{n}, p\right)
\end{gathered}
$$

3 material balances

$$
\frac{\partial \dot{n}_{o}^{x}}{\partial x}+\frac{\partial \dot{n}_{o}^{y}}{\partial y}=0
$$

condition for total pressure

$$
p=p_{o}+p_{w}+p_{n}
$$

Tafel equation for current density $i$ on reaction layer

$$
\begin{aligned}
& i=f_{v} i_{0}\left(\frac{p_{o}}{p_{o}^{r e f}}\right)^{\gamma} \exp \left[\frac{\alpha n F\left(U_{0}-U_{z}-\frac{d_{m e m}}{\kappa_{m e m}} i\right)}{R T}\right] \\
& i=0 \text { in the remainder (dummy variable) }
\end{aligned}
$$




\section{Numerical Simulation of PEM Fuel Cells (continued)}

Contour plot of molar flux density of water vapour in y-direction and its error (typical result)
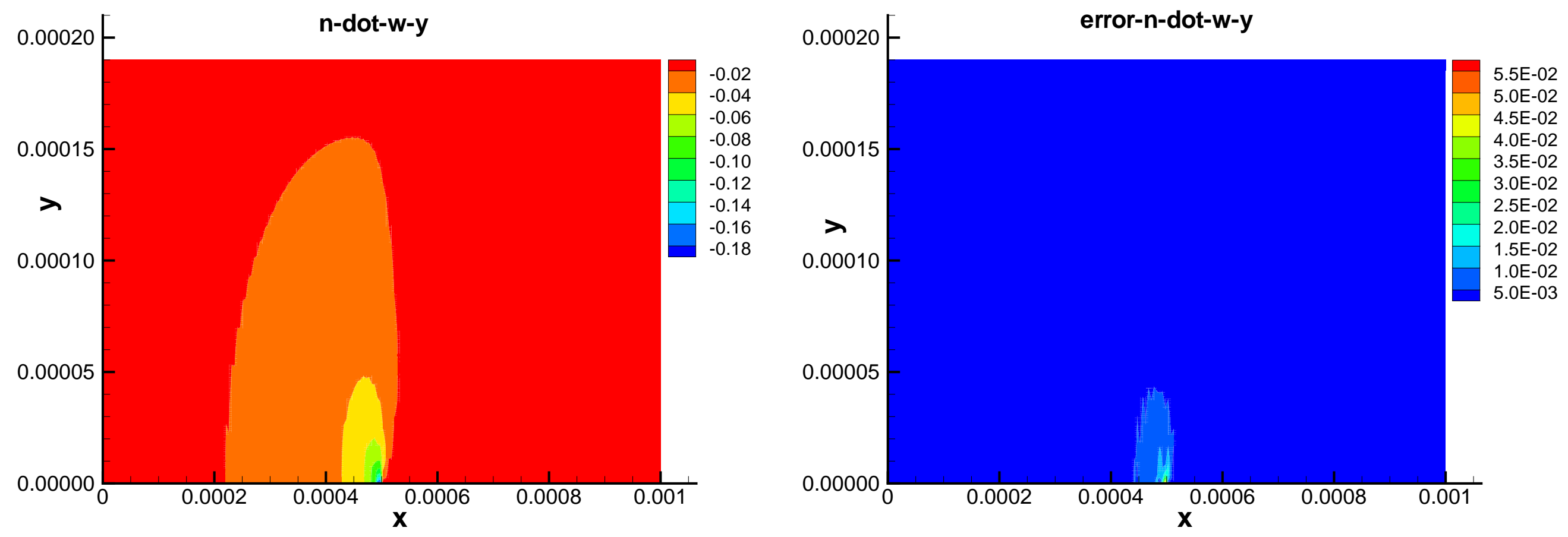


\section{Forschungszentrum Karlsruhe}

in der Helmholtz-Gemeinschaft

\section{Numerical Simulation of PEM Fuel Cells (continued)}

Current density along the reaction layer and its error

$i_{\text {mean }}=2981,92$

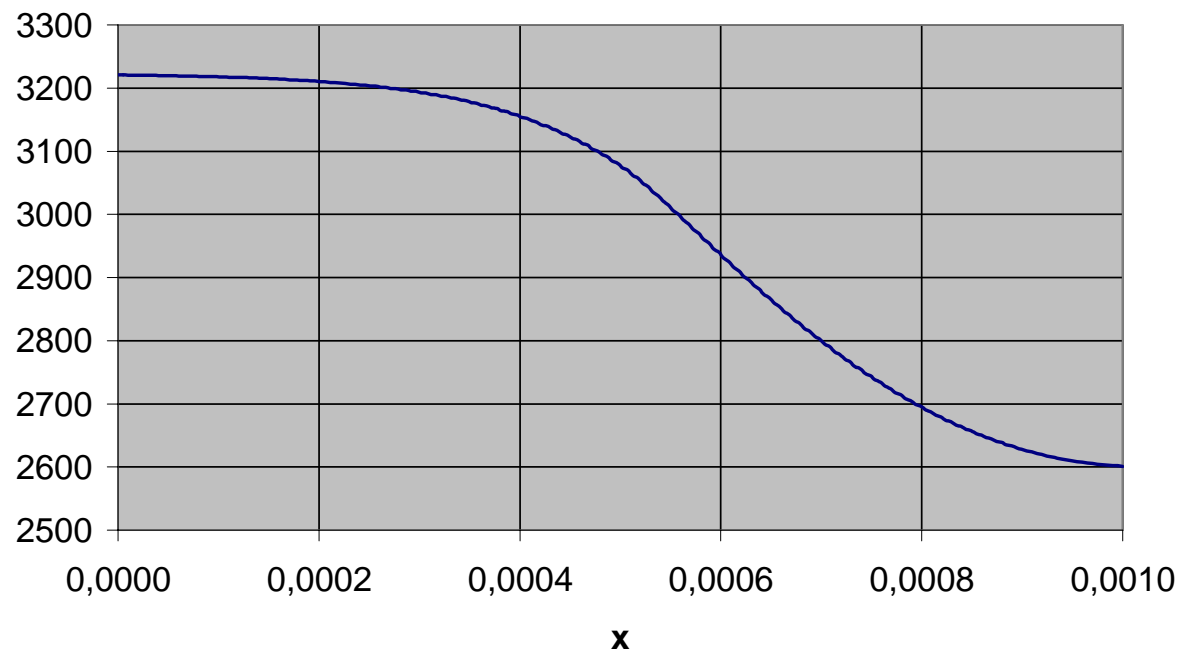

error-i

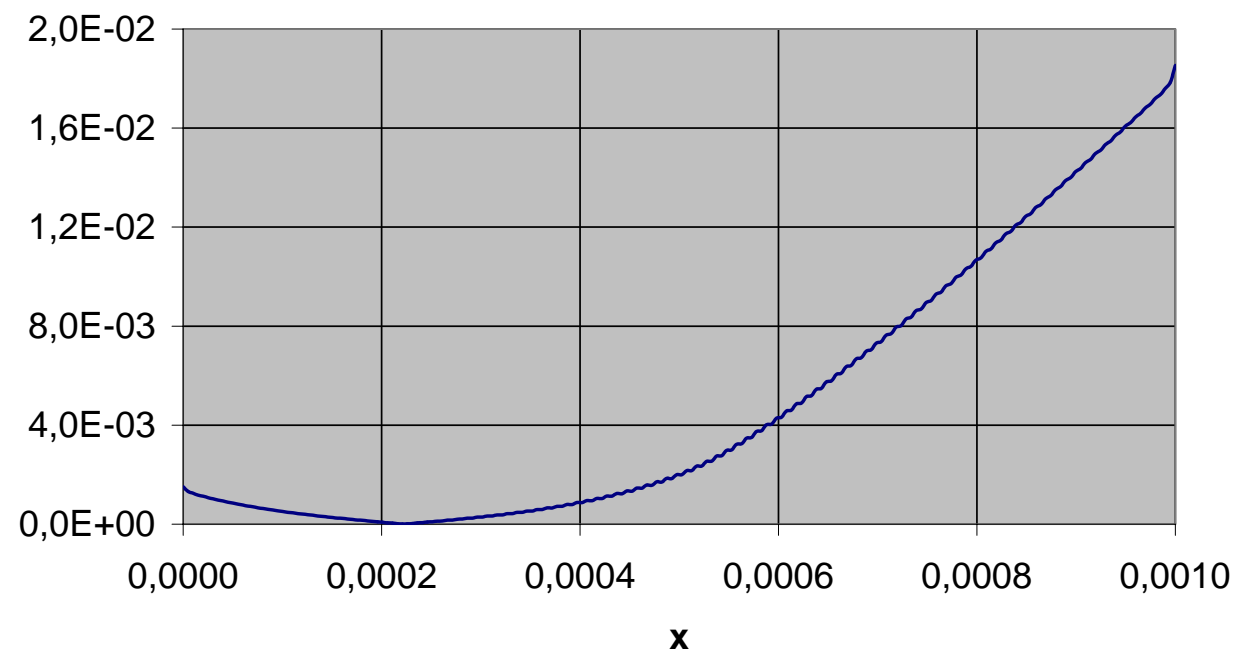




\section{Forschungszentrum Karlsruhe}

in der Helmholtz-Gemeinschaft

\section{Numerical Simulation of Solid Oxide Fuel Cells}

Solution domain consists of 2 subdomains: Anode and gas channel

Dividing line, coupling conditions, different PDE systems

Variables: Flow velocities in $\mathrm{x}$ - and $\mathrm{y}$-direction, mole fractions for methane, carbon monoxide, hydrogen, carbon dioxide and steam, and pressure

Nonlinear system of 8 PDEs

Rectangular grids: $80 \times 41$ in anode and gas channel

Consistency order $\mathrm{q}=4$

HP XC6000 with 8 processors

CPU time for master processor: $510 \mathrm{sec}$

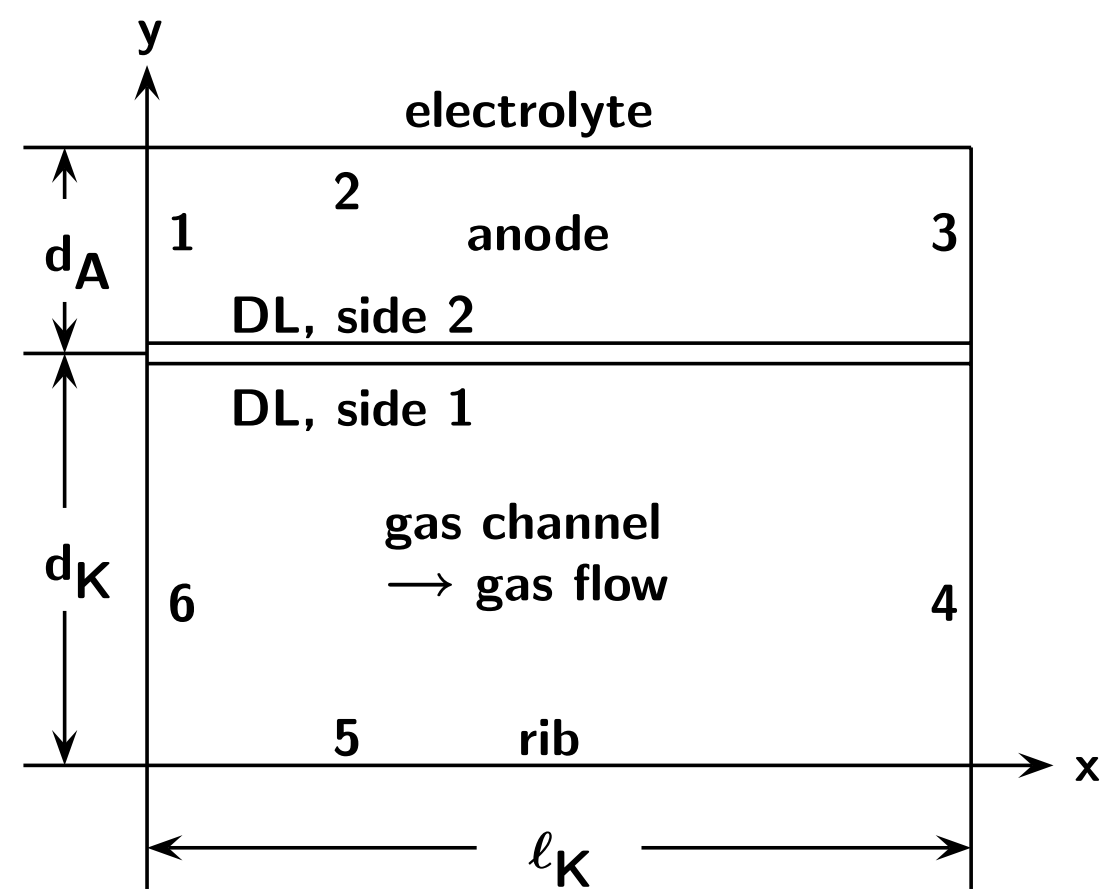




\section{Forschungszentrum Karlsruhe} in der Helmholtz-Gemeinschaft

\section{Numerical Simulation of Solid Oxide Fuel Cells (continued)}

\section{Gas channel}

4 species balances

$$
-\left[\frac{\partial\left(p_{K} \cdot u_{x, K} \cdot Y_{C O, K}\right)}{\partial x}+\frac{\partial\left(p_{K} \cdot u_{y, K} \cdot Y_{C O, K}\right)}{\partial y}\right]+\frac{\partial\left(D_{C O, g a s} \frac{\partial\left(p_{K} \cdot Y_{C O, K}\right)}{\partial y}\right)}{\partial y}=0
$$

Equation of continuity

$$
\frac{\partial\left(\rho_{K} \cdot u_{x, K}\right)}{\partial x}+\frac{\partial\left(\rho_{K} \cdot u_{y, K}\right)}{\partial y}=0
$$

2 Navier-Stokes equations

$$
\rho_{K} \cdot\left(u_{x, K} \frac{\partial u_{x, K}}{\partial x}+u_{y, K} \frac{\partial u_{x, K}}{\partial y}\right)=-\frac{\partial p_{K}}{\partial x}+\frac{\partial}{\partial x}\left(\mu\left(2 \cdot \frac{\partial u_{x, K}}{\partial x}-\frac{2}{3} \cdot\left(\frac{\partial u_{x, K}}{\partial x}+\frac{\partial u_{y, K}}{\partial y}\right)\right)\right)+\frac{\partial}{\partial y}\left(\mu\left(\frac{\partial u_{x, K}}{\partial y}+\frac{\partial u_{y, K}}{\partial x}\right)\right)
$$

Dalton's law

$$
Y_{3}+Y_{4}+Y_{5}+Y_{6}+Y_{7}=1
$$




\section{Forschungszentrum Karlsruhe} in der Helmholtz-Gemeinschaft

\section{Numerical Simulation of Solid Oxide Fuel Cells (continued)}

\section{Anode}

5 species balances

$$
-\left[\frac{\partial\left(p_{A} \cdot u_{x, A} \cdot Y_{C O, A}\right)}{R T \partial x}+\frac{\partial\left(p_{A} \cdot u_{y, A} \cdot Y_{C O, A}\right)}{R T \partial y}\right]+\frac{\partial\left(D_{C O, g a s}^{e f f} \frac{\partial\left(p_{A} \cdot Y_{C O, A}\right)}{R T \partial y}\right)}{\partial y}+\frac{\partial\left(D_{C O, g a s}^{e f f} \frac{\partial\left(p_{A} \cdot Y_{C O, A}\right)}{R T \partial x}\right)}{\partial x}+r_{C H_{4}}-r_{s}=0
$$

Darcy's law in $\underline{x}$ - and $y$-direction

$$
\frac{\partial p_{A}}{\partial x}=-\frac{\mu}{k_{p}} u_{x, A}
$$

Dalton's law

$$
Y_{3}+Y_{4}+Y_{5}+Y_{6}+Y_{7}=1
$$




\section{Numerical Simulation of Solid Oxide Fuel Cells (continued)}

Mole fraction of carbon monoxide and its error for the anode
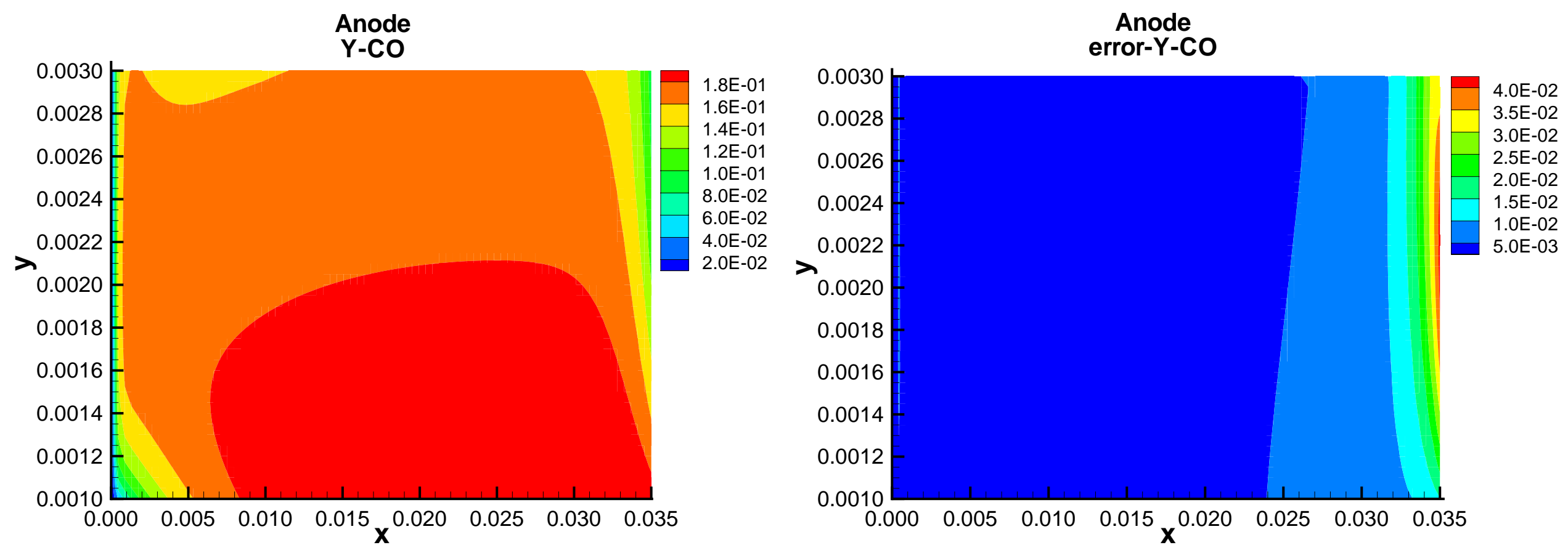


\section{Fluid/Structure Interaction for a High Pressure Diesel Injection Pump}

Solution domain consists of 3 subdomains: Housing, piston and lubrication gap

Dividing lines, coupling conditions, different PDE systems

Variables: displacements in $\mathrm{z}$ - and r-direction and stresses in housing and piston velocities in $\mathbf{z}$ - and $\mathbf{r}$-direction and pressure in lubrication gap

Nonlinear system of 6 PDEs in housing and piston 3 PDEs in gap

Rectangular grids: $401 \times 81$ in housing $401 \times 641$ in gap $401 \times 40$ in piston

Consistency order $q=2$

CPU time for master processor:

HP XC6000, 32 proc., Itanium2 $1.5 \mathrm{GHz}$, Quadrics interconnect:

$452.5 \mathrm{~min}$ cf. SGI Altix $\mathbf{4 7 0 0}$ (LRZ Munich), 32 proc., Itanium2 $1.6 \mathrm{GHz}$, NUMAlink:

$385.5 \mathrm{~min}$
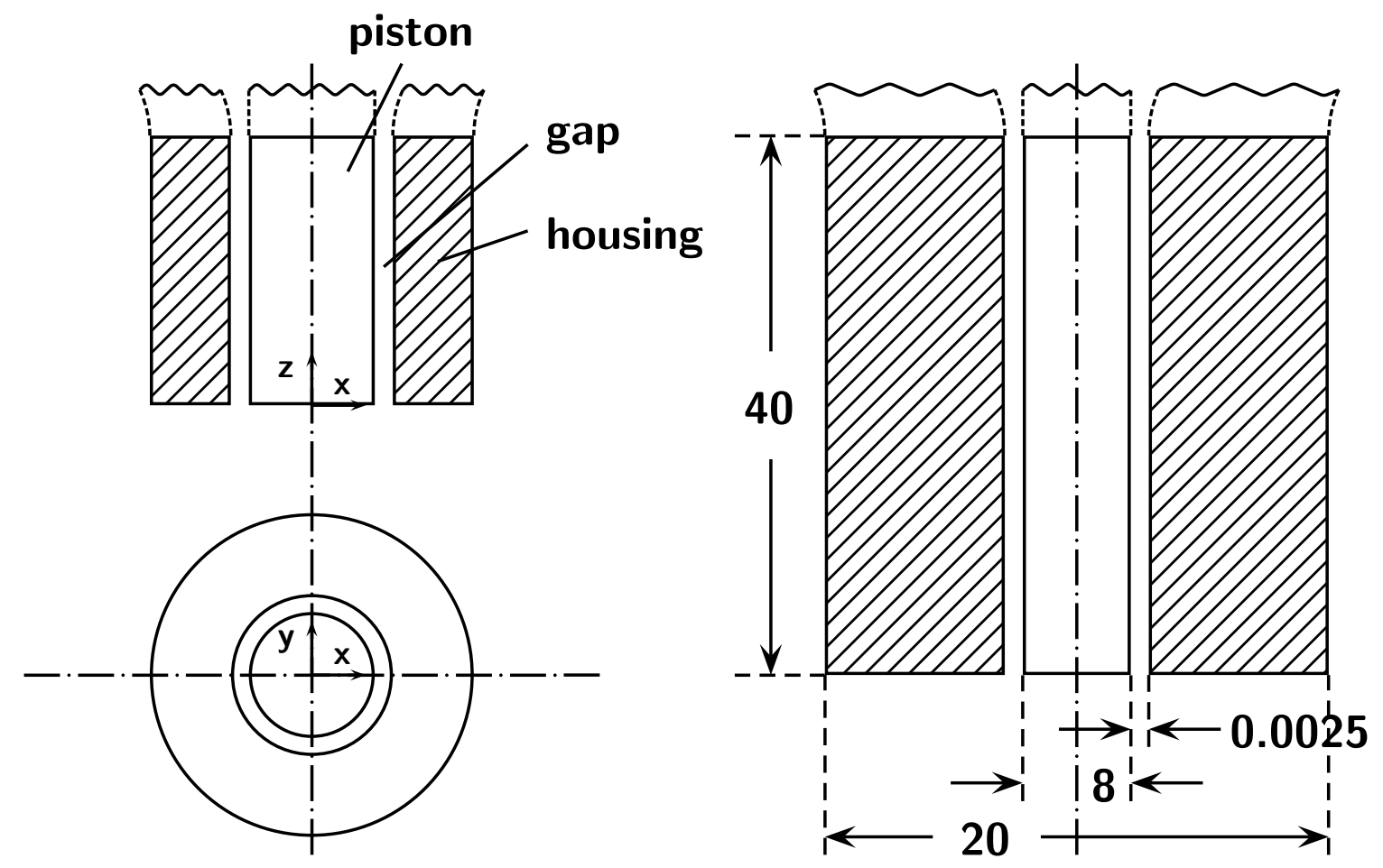


\section{Forschungszentrum Karlsruhe}

in der Helmholtz-Gemeinschaft

\section{Fluid/Structure Interaction for a High Pressure Diesel Injection Pump (continued)}

\section{Piston/Housing}

3 elasticity equations in $z_{-}, \varphi-, r$-direction

$$
\frac{1}{E}\left[\sigma_{z}-\sigma_{z, \text { old }}-\nu\left(\sigma_{\varphi}-\sigma_{\varphi, \text { old }}\right)-\nu\left(\sigma_{r}-\sigma_{r, \text { old }}\right)\right]-\frac{\partial w}{\partial z}=0
$$

1 elasticity equation in rz-direction

$$
\frac{1+\nu}{E}\left(\tau_{r z}-\tau_{r z, o l d}\right)-\frac{1}{2}\left(\frac{\partial u}{\partial z}+\frac{\partial w}{\partial r}\right)=0
$$

2 equilibrium equations

$$
\frac{\partial \sigma_{r}}{\partial r}+\frac{\partial \tau_{r z}}{\partial z}+\frac{1}{r}\left(\sigma_{r}-\sigma_{\varphi}\right)=0
$$

\section{Lubrication gap}

2 Navier-Stokes equations

$$
u \frac{\partial u}{\partial r}+w \frac{\partial u}{\partial z}=-\frac{1}{\rho} \frac{\partial p}{\partial r}+\frac{\eta}{\rho}\left(\frac{\partial^{2} u}{\partial r^{2}}+\frac{1}{r} \frac{\partial u}{\partial r}-\frac{u}{r^{2}}+\frac{\partial^{2} u}{\partial z^{2}}\right)
$$

Equation of continuity

$$
\frac{\partial u}{\partial r}+\frac{u}{r}+\frac{\partial w}{\partial z}=0
$$




\section{Forschungszentrum Karlsruhe}

in der Helmholtz-Gemeinschaft

\section{Fluid/Structure Interaction for a High Pressure Diesel Injection Pump (continued)}

Nested iterations for the solution process necessary

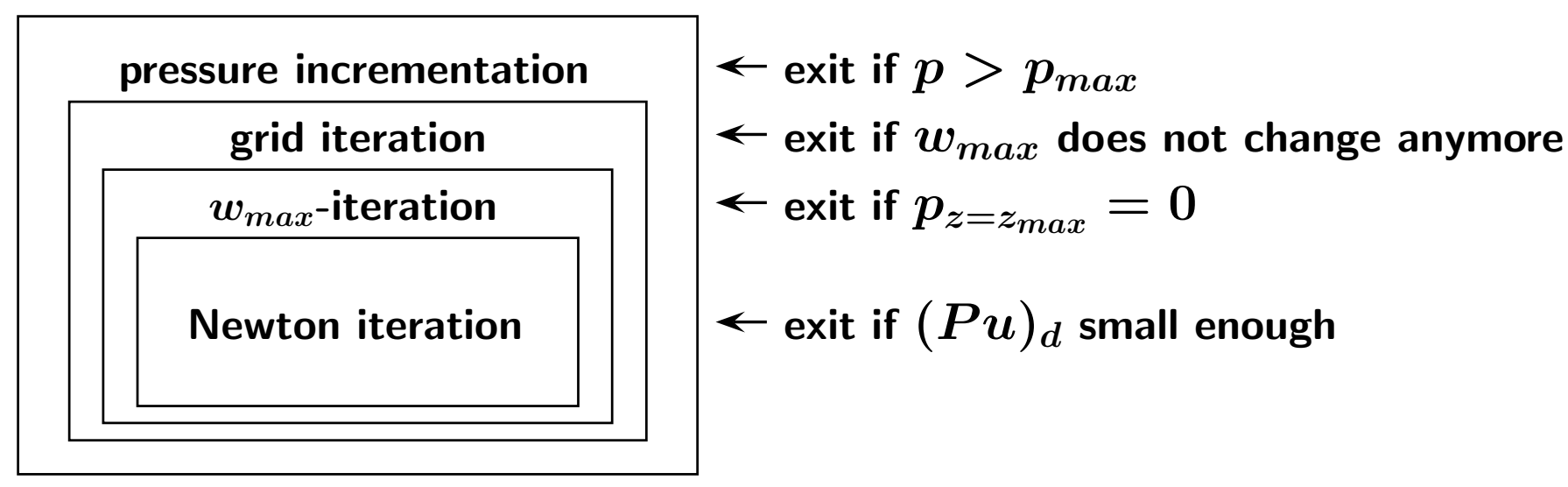




\section{Fluid/Structure Interaction for a High Pressure Diesel Injection Pump (continued)}

Velocity $w$ in z-direction for 2000 bar and its error
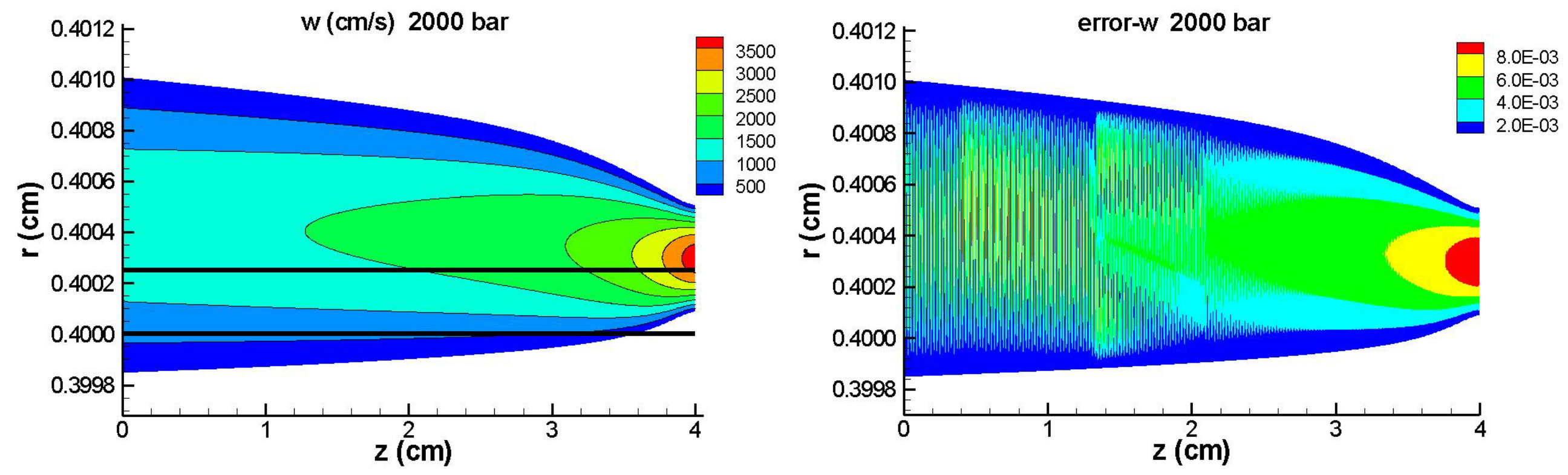

Volume flow: $2.65 \mathrm{~cm}^{3} / \mathrm{s}$ 


\section{Forschungszentrum Karlsruhe}

in der Helmholtz-Gemeinschaft

\section{Concluding Remarks}

- Black-box PDE solver FDEM (URL: http://www.rz.uni-karlsruhe.de/rz/docs/FDEM/Literatur)

- User input: any PDE system, any domain, 2-D and 3-D

- Domain may consist of several subdomains with different PDEs $\rightarrow$ Dividing lines

- Unique feature: error estimate

- Efficient parallelization with MPI

We offer a service to solve the PDEs of cooperation partners. 